\title{
Genetic parameter estimates for growth traits of Large White pigs in Kenya
}

\author{
E.D. Ilatsia $^{1 \#}$, M.G. Githinji ${ }^{1}$, T.K. Muasya ${ }^{1}$, T.O. Okeno ${ }^{1}$ and A.K. Kahi ${ }^{2}$ \\ ${ }^{1}$ Animal Genetic Resources Group, National Animal Husbandry Research Centre, KARI-Naivasha, \\ P.O. Box 25, Naivasha 20117, Kenya \\ ${ }^{2}$ Animal Genetics and Breeding Group, Department of Animal Sciences, Egerton University, \\ P.O. Box 536, Egerton 20115, Kenya
}

\begin{abstract}
Variance components and genetic parameter were estimated for piglet growth traits of Large White (LW) pigs managed under intensive production systems in Kenya. Data were on piglet performance of LW pigs born between 1982 to 1996 . Growth performance traits of the piglets that were considered, were body weight (BW, in kg) at birth (BW0), three weeks (BW3), eight weeks at weaning (BW8), 12 weeks ( BW12), 24 weeks (BW24) and 36 weeks (BW36); and average daily gain (g/day) from birth to eight weeks ( $\left.\mathrm{DG}_{0-8}\right)$ and from weaning to 24 weeks $\left(\mathrm{DG}_{8-24}\right)$. Variance components and genetic parameters were estimated using univariate and multivariate animal models. Direct heritability estimates from univariate analyses were 0.38 , $0.24,0.47,0.39,0.36$ and 0.26 for BW0, BW3, BW8, BW12, BW24 and BW36, respectively. Maternal heritabilities were $0.42,0.250 .15$ and 0.18 for BW0, BW3, BW8 and $\mathrm{DG}_{0-8}$, respectively. Genetic correlations among growth traits were positive and ranged from 0.45 to 0.98 while phenotypic correlations ranged from 0.54 to 0.95 . Early piglet growth was highly heritable and under the influence of maternal effects. Selection programmes for genetic improvement of early growth performance should be based on models that account for direct and maternal genetic and litter effects.
\end{abstract}

Keywords: Additive genetic effects, body weight, swine, litter effects, maternal effects

${ }^{\#}$ Corresponding author. E-mail: evansilatsia@yahoo.com

\section{Introduction}

Pig production in Kenya is practiced mainly as intensive and semi intensive smallholder systems, and large scale commercial enterprises (Wanjaiya, 1999; Githinji et al., 2007) where the Large White (LW) is the main breed utilised due to its desirable growth potential and high fecundity. The main challenge to improved pig productivity in Kenya and other developing countries is lack of organised breeding programmes to facilitate genetic improvement (Pathiraja, 1987; Kahi et al., 2006; Chimonyo \& Dzama, 2007). This is mainly because the important components for design of breeding programmes such as definition of breeding objectives, choice of selection criteria, genetic evaluations, selection and design of appropriate mating systems and strategies for the dissemination of genetic superiority are missing (Kahi et al., 2006). Furthermore, genetic improvement programmes can only be effectively implemented where accurate performance records on traits of economic importance and reliable pedigree information are available.

In pig production, growth and reproductive traits are important (Crump et al., 1997a; Mungate et al., 1999; Hermesch et al., 2000a; Zhang et al., 2000). Therefore, to effectively design appropriate improvement programmes, performance evaluation of these traits using appropriate models becomes critical. Environment specific estimates obtained by appropriate analytical models are usually desirable (Bourdon, 1998; Crump et al., 1997a) and would be more efficient when applied in tropical pig breeding programmes. However, as a result of limitations in performance records, accurately quantified performance parameter estimates for economically important traits in pig breeds in developing countries are scarce.

Kabare (1991) presented genetic parameters for growth and reproductive traits of various European pig breeds in Kenya. In that study it was assumed that there was no relationship between the dams and that the sires were mated randomly to the dams. This assumption does not hold in most pig breeding programmes because sires are usually assigned to particular dams. Furthermore, maternal and common litter effects were ignored. Ignoring maternal and litter effects would result in overestimation of genetic parameters and consequently inaccurate predictions of response to selection (Hermesch et al., 2000b; Zhang et al., 2000; Solanes et al., 2004; Ehlers et al., 2005). The most reliable way of making use of performance information in 
selection is through use of appropriate models in prediction of the genetic merit of individual animals. Such information is important in designing appropriate management and breeding strategies aimed at improving productivity. The objective of this study was to estimate variance components and genetic parameters for growth traits of LW pigs reared under intensive management systems in Kenya.

\section{Materials and Methods}

Data were obtained from the pig research unit at the National Animal Husbandry Research Centre (NAHRC), Naivasha, Kenya. The NAHRC is located approximately $70 \mathrm{~km}$ northwest of Nairobi at an altitude of 1829 - 2330 metres above sea level in agro-ecological zone IV, classified as semi-arid. Data on piglet performance of LW pigs born between 1982 to 1996 were extracted from the available performance sheets kept at the Pig Research Unit. Piglets' growth performance traits considered, were body weight (BW, in $\mathrm{kg}$ ) at birth (BW0), three weeks (BW3), eight weeks at weaning (BW8), 12 weeks ( BW12), 24 weeks (BW24) and 36 weeks (BW36); and average daily gain (g/day) from birth to eight weeks $\left(\mathrm{DG}_{0-8}\right)$ and from weaning to 24 weeks $\left(\mathrm{DG}_{8-24}\right)$. Individual litter BW0 was recorded within the first 24 hours of birth. Litter size at birth included only piglets that were alive after the first 24 hours of birth. Based on sow and litter feeding management over the years, animals were assigned to two management groups (MG). In the first MG (from 1982 to 1989), sows were fed a sow and weaner meal $(140 \mathrm{~g} \mathrm{CP} / \mathrm{kg}$ ) at a rate of $3 \mathrm{~kg}$ per day and an additional $0.25 \mathrm{~kg}$ per piglet suckling. The daily ration was divided into equal meals provided in the morning and afternoon. Piglets were confined in the creep area where a creep feed was provided ad libitum. They were allowed to suckle three times a day. After weaning up to 18 weeks of age the piglets were fed a sow and weaner meal according to body weight. After 18 weeks they received a pig finisher meal. In the second MG (from 1990 to 1996) the sows were fed $2 \mathrm{~kg}$ of a sow and weaner meal divided between the morning and evening. No additional feed was provided per suckling piglet. Piglets stayed with their dam up to weaning and received a restricted allowance of a creep feed, depending on litter size. At weaning piglets in litters were separated and allocated to group pens, depending on sex and weaning weight. Piglets were fed a sow and weaner meal depending on their individual weights until they attained the mature sale weight at ca. 36 weeks of age. Regular de-worming, spraying to control ecto-parasites and vaccinations against notifiable diseases were the same for both MGs. Data were edited to eliminate all records from sows with unknown parity and litter size. The structure and a summary of the statistics of the data used in the final analyses are shown in Table 1. The rather high CV could be attributed to some variations in herd management within MG, and also the mixing of castrated and intact males into one group. These would result in variations in growth performance as well. However, such differences were difficult to discern from the available records at the Pig Research Unit.

Table 1 Data structure and summary statistics for growth traits

\begin{tabular}{lrrrrrrrr}
\hline \multirow{2}{*}{ Item } & \multicolumn{10}{c}{ Traits $^{\mathrm{a}}$} \\
\cline { 2 - 9 } & BW0 & BW3 & BW8 & BW12 & BW24 & BW36 & DG $_{0-8}$ & DG $_{8-24}$ \\
\hline Data structure & & & & & & & & \\
$\quad$ Number of animal & 2108 & 1841 & 1647 & 1472 & 1109 & 969 & 1647 & 1109 \\
$\quad$ Sires with progeny & 28 & 28 & 25 & 21 & 21 & 19 & 28 & 25 \\
$\quad$ Dams with progeny & 158 & 158 & 158 & 147 & 141 & 139 & 158 & 141 \\
Summarized statistics & & & & & & & & \\
$\quad$ Units & $\mathrm{kg}$ & $\mathrm{kg}$ & $\mathrm{kg}$ & $\mathrm{kg}$ & $\mathrm{kg}$ & $\mathrm{kg}$ & $\mathrm{g} / \mathrm{day}$ & $\mathrm{g} / \mathrm{day}$ \\
Mean & 1.5 & 4.9 & 11.0 & 15.0 & 42.7 & 75.7 & 196.1 & 381.7 \\
s.d. & 0.5 & 1.7 & 3.3 & 4.5 & 10.4 & 15.1 & 58.0 & 92.8 \\
C.V. (\%) & 33.3 & 34.7 & 30.0 & 30.0 & 24.4 & 19.6 & 29.6 & 24.3
\end{tabular}

\footnotetext{
${ }^{\mathrm{a}}$ Traits: BW0 - body weight at birth; BW3 - weight at three weeks; BW8 - weight at eight weeks, at weaning;

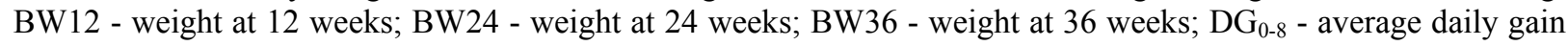
form birth to eight weeks; $\mathrm{DG}_{8-24}$ - average daily gain from weaning to 24 weeks.
} 
Preliminary analyses to determine fixed effects to be included in subsequent mixed models were performed using analysis of variance (SAS, 1998). Table 2 shows the relevant fixed effects for the traits studied as well as the corresponding levels of significance and proportions of the variation explained by these effects $\left(\mathrm{R}^{2}\right)$. Dam parity was included in the model and classified into five classes, consisting of the first three parities, the forth and fifth parities grouped together, and parities higher than the fifth.

Table 2 Structure of the models used in the final analyses, levels of significance and proportion of variation explained by the models $\left(\mathrm{R}^{2}\right)$

\begin{tabular}{llllllllll}
\hline \multirow{2}{*}{ Factors } & Type $^{\mathrm{a}}$ & \multicolumn{7}{c}{ Trait $^{\mathrm{b}}$} \\
\cline { 3 - 9 } & & BW0 & BW3 & BW8 & BW12 & BW24 & BW36 & DG $_{0-8}$ & DG $_{8-24}$ \\
\hline MG & $\mathrm{F}$ & $* * *$ & $* * *$ & $* * *$ & $* * *$ & $* * *$ & $* * *$ & $* * *$ & $* * *$ \\
Dam parity & $\mathrm{F}$ & $* * *$ & $* * *$ & $* * *$ & $* * *$ & $* * *$ & $* * *$ & $* * *$ & $* * *$ \\
Sex & $\mathrm{F}$ & $* *$ & $* * *$ & $* * *$ & $* * *$ & $* * *$ & $* * *$ & $* *$ & $* * *$ \\
Litter size & $\mathrm{C}$ & $* * *$ & $* * *$ & $* *$ & $* *$ & $* *$ & $* *$ & $* * *$ & $* *$ \\
BW(0) & $\mathrm{C}$ & & $* * *$ & $* * *$ & $* * *$ & $* *$ & $* *$ & $* *$ & $* * *$ \\
$\mathrm{R}^{2}$ & $\mathrm{~A}$ & 0.35 & 0.26 & 0.22 & 0.18 & 0.24 & 0.29 & 0.22 & 0.23 \\
Direct effect & $\mathrm{V}$ & $\sqrt{ }$ & $\sqrt{ }$ & $\sqrt{ }$ & $\sqrt{ }$ & $\sqrt{ }$ & $\sqrt{ }$ & $\sqrt{ }$ & $\sqrt{ }$ \\
Maternal effect & $\mathrm{A}$ & $\sqrt{ }$ & $\sqrt{ }$ & $\sqrt{ }$ & $\sqrt{ }$ & $\sqrt{ }$ & $\sqrt{ }$ & $\sqrt{ }$ & $\sqrt{ }$ \\
Litter effect & $\mathrm{R}$ & & & & & & & & \\
& & & & & & & & &
\end{tabular}

$* * * \mathrm{P}<0.001 ; * * \mathrm{P}<0.01$

$\mathrm{MG}$ - management group.

${ }^{a}$ Type of factors: F - fixed factor; C - linear covariable; R - random factor with identity matrix; A random factor with numerator relationship matrix.

${ }^{\mathrm{b}}$ See Table 1 for description of traits.

Genetic analyses were performed based on derivative free restricted likelihood methodology (Meyer, 1989). Mixed model equations in the analyses were solved iteratively and estimates from previous runs were used as starting values for the subsequent runs until no differences were observed in variance components in at least two consecutive runs. Preliminary analyses showed that the best model (based on maximum log likelihood) that fitted the data was a model with three random effects, i.e. direct additive genetic, maternal genetic and litter environmental effects. Table 2 shows the various random effects fitted for each trait in the univariate analyses. The data structure (number of offspring per dam) and the number of dams and grand dams with records are important determinants in estimating maternal effects (Meyer et al., 1992; Hermesch et al., 2000a; Maniatis \& Pollot, 2003; Solanes et al., 2004). Due to limitations in the pedigree and performance data structure (Table 1), maternal environment effects and covariance between maternal and direct genetic effects were not considered in the models to estimate variance components. Different univariate models including combinations of direct and maternal genetic effects and litter (due to common litter environment) were fitted. The general mixed model fitted was:

$$
y=X b+Z_{a} a+Z_{m} m+Z_{c} c+e \operatorname{cov}(a, m)=0
$$

Model 1

where $y$ is the observation on piglet growth traits; $X$ and $Z$ are incidence matrices relating observation to the fixed and random effects, respectively; $b, a, m c$ and $e$ are vectors of fixed, direct additive genetic, maternal genetic, common litter and random residual effects, respectively. As alluded to earlier, maternal effects were not fitted in the analyses of BW12, BW24, BW36 and DG 8 -24. A multitrait model similar to Model 1 was fitted to estimate genetic and phenotypic correlation among the six body weight measures. The effects fitted in this model were similar to those fitted in Model 1 but the maternal and common litter effects were ignored. The phenotypic variances were defined as $\sigma_{a}^{2}+\sigma_{m}^{2}+\sigma_{c}^{2}+\sigma_{e}^{2}$. The direct heritability $\left(\mathrm{h}^{2}\right)$, 
maternal heritability $\left(\mathrm{m}^{2}\right)$ and the litter environmental effect $\left(\mathrm{c}^{2}\right)$ were computed as $\sigma_{a}^{2} / \sigma_{p}^{2}, \sigma_{m}^{2} / \sigma_{p}^{2}$, and $\sigma_{c}^{2} / \sigma_{p}^{2}$, respectively. Estimates at least two times higher than their corresponding standard error were assumed to be significantly different from zero. The random effects were assumed to follow a normal distribution with mean zero and covariance structure:

$$
\operatorname{var}\left[\begin{array}{c}
a \\
m \\
c \\
e
\end{array}\right]=\left[\begin{array}{cccc}
A \sigma_{a}^{2} & 0 & 0 & 0 \\
0 & A \sigma_{m}^{2} & 0 & 0 \\
0 & 0 & I \sigma_{c}^{2} & 0 \\
0 & 0 & 0 & I \sigma_{e}^{2}
\end{array}\right]
$$

where $a$ is a vector of additive genetic effects; $m$ is a vector of maternal genetic effects; $c$ is a vector of random environmental litter effects; $e$ is a vector of random residual effects; $A$ is the numerator relationship matrix; $I$ is the identity matrix; $\sigma_{\mathrm{a}}^{2}$ is the direct additive genetic variance; $\sigma_{\mathrm{m}}^{2}$ is the variance due maternal genetic effects; $\sigma_{c}^{2}$ is variance for environmental litter effects; $\sigma^{2}{ }_{\mathrm{e}}$ is the residual variance.

\section{Results and Discussion}

The estimates of variance components and genetic parameter estimates from univariate analyses are summarized in Table 3. Maternal heritability estimates were reported only for pre-weaning piglet growth traits. The common litter environmental effects were high for BW0, BW3, BW8 and $\mathrm{DG}_{0-8}$ but low and nonsignificant for post-weaning growth traits (Table 3). Maniatis \& Pollot (2002) and Solanes et al. (2004) reported reduced importance of litter environmental effects with advanced age for growth traits of lambs and Yorkshire pigs, respectively. During the post-weaning period in this study, piglets from the same litter were separated in different pens depending on sex and weight at weaning, which could have resulted in nonsignificant litter effects. The magnitude of the common litter effects reported in this study and in other studies (e.g. Zhang et al., 2000; Hermesch et al., 2000a; Solanes et al., 2004) indicated that common litter effects should be included in models for genetic evaluation and prediction of breeding value for early growth performance of piglet. Ignoring litter environmental effects in evaluation of early growth performance would lead to biased breeding value estimates and over-prediction of the potential genetic gain expected from a pig improvement programme. Models that ignore common litter effects could be considered in evaluation of post-weaning growth traits.

Table 3 Variance component and genetic parameter estimates for growth traits from univariate analyses

\begin{tabular}{lllllllll}
\hline Estimates $^{\mathrm{b}}$ & \multicolumn{8}{c}{ Trait $^{\mathrm{a}}$} \\
\cline { 2 - 9 } & BW0 & BW3 & BW8 & BW12 & BW24 & BW36 & DG $_{0-8}$ & DG $_{8-24}$ \\
\hline$\sigma^{2}{ }^{2}$ & 0.18 & 0.47 & 8.17 & 8.74 & 69.41 & 72.35 & 2919.78 & 6802.65 \\
$\sigma^{2}$ & 0.20 & 0.50 & 2.56 & & & & 1115.00 & \\
$\sigma^{2}{ }^{2}$ & 0.13 & 0.58 & 2.57 & 1.66 & 10.99 & 14.40 & 948.42 & 1606.00 \\
$\sigma^{2}$ & 0.48 & 2.00 & 17.40 & 22.13 & 195.36 & 275.02 & 6272.78 & 16269.00 \\
$\mathrm{~h}^{2}$ & $0.38 \pm 0.10$ & $0.24 \pm 0.08$ & $0.47 \pm 0.10$ & $0.39 \pm 0.10$ & $0.36 \pm 0.09$ & $0.26 \pm 0.10$ & $0.46 \pm 0.10$ & $0.40 \pm 0.10$ \\
$\mathrm{~m}^{2}$ & $0.42 \pm 0.11$ & $0.25 \pm 0.09$ & $0.15 \pm 0.07$ & & & & $0.18 \pm 0.07$ & \\
$\mathrm{c}^{2}$ & $0.27 \pm 0.08$ & $0.29 \pm 0.08$ & $0.15 \pm 0.06$ & $0.07 \pm 0.05$ & $0.06 \pm 0.04$ & $0.05 \pm 0.04$ & $0.15 \pm 0.04$ & $0.09 \pm 0.05$
\end{tabular}

${ }^{\mathrm{a}}$ See Table 1 for description of traits.

${ }^{\mathrm{b}}$ Estimates: $\sigma_{\mathrm{a}}^{2}$ - direct additive genetic variance; $\sigma_{\mathrm{m}}^{2}$ - maternal genetic variance; $\sigma^{2}{ }_{\mathrm{c}}$ - common litter environmental variance; $\sigma_{p}^{2}$ - phenotypic variance; $\mathrm{h}^{2}$ - direct heritability; $\mathrm{m}^{2}$ - maternal heritability; $\mathrm{c}^{2}$ - ratio of litter environmental variance to total variance. 
Maternal heritability for pre-weaning piglet growth performance ranged from 0.15 to 0.42 and progressively reduced up to weaning (Table 3$)$. Direct heritability estimates for BW0 (0.38) and BW3 (0.24) and the maternal heritability estimate for BW0 $(0.42)$ reported in this study were higher than similar estimates reported in the literature. This could be expected because of differences in the structure and amount of data and also in the models fitted. As alluded earlier, the amount and structure of the data could not allow variation arising from maternal environment and the interaction between additive genetic and maternal genetic effect to be estimated effectively. In this case it is possible that variations attributable to these effects would be apportioned to the additive genetic effect resulting in higher estimates than would be expected. As more data are made available from existing pig herds in the country, these parameters could be estimated using models that would appropriately partition the phenotypic variance more accurately. Solanes et al. (2004) reported a non-significant direct heritability of 0.03 and a maternal heritability of 0.17 at three weeks of age. Similarly, Solió et al. (1994) reported direct heritability estimates of between 0.0 to 0.08 and maternal heritability estimates of between 0.16 to 0.17 in several lines of Iberian pigs. In Australian pigs, Hermesch et al. (2000b) reported direct and maternal heritability estimates of 0.04 and 0.13 , respectively, for weight at two weeks of age. In Chinese-European Tiemelsan composites, Zhang et al. (2000) reported direct and maternal heritability estimates of 0.03 and 0.11 , respectively, for individual weight of piglets at one month of age. The magnitude of the maternal genetic variance and the resultant maternal heritability suggest that the pre-weaning growth of piglet is largely influenced by both maternal and direct additive genetic effects which is in agreement with these findings.

Direct additive genetic effects were the main random effect influencing post-weaning piglet performance. Moderate to high direct heritability estimates were reported for post-weaning growth traits (BW12, BW24, BW36 and $\mathrm{DG}_{8-24}$ ). These estimates and the importance of direct additive genetic effects and the corresponding less significance of maternal genetic effects with age agree well with results presented in the literature (e.g. Hermesch et al., 2000b; Zhang et al., 2000; Solanes et al., 2004; Chimonyo \& Dzama, 2007) but disagree with Johnson et al. (2002) who reported moderate maternal heritability for post-weaning piglet growth performance. In this study, maternal genetic effects were insignificant for post-weaning growth performance, in agreement with reports in the literature (e.g. Crump et al., 1997a; Zhang et al., 2000; Solanes et al., 2004). However, it should be noted that maternal effects could be estimated more accurately using large data sets with a better structure to explain some of the phenotypic variation more adequately. In this study, however, the numbers of dams and grand dams with records were few (Table 1). It has been noted that such kind of data structure makes the estimation of maternal effects and the correlation between maternal and direct additive effects delicate (Meyer, 1992; Hermesch et al., 2000b; Maniatis \& Pollot, 2003; Solanes et al., 2004).

The estimates of direct heritability for $\mathrm{DG}_{0-8}$ and $\mathrm{DG}_{8-24}$ in this study were within the range of literature estimates. Summarised literature results for post-weaning daily gain range from 0.14 to 0.76 with a mean estimate of 0.30 (Clutter \& Brascamp, 1998). Solanes et al. (2004) reported a direct heritability of 0.43 and 0.32 for weight gain from birth to $90 \mathrm{~kg}$ and from $25 \mathrm{~kg}$ body weight to $90 \mathrm{~kg}$, respectively. In another study involving the indigenous Mukota breed in Zimbabwe, Chimonyo \& Dzama, (2007) reported heritability estimates of 0.15 and 0.27 and maternal heritabilities of 0.2 and 0.03 for pre-weaning and post-weaning daily gains, respectively. Solanes et al. (2004) used a model that assumed additivity of all genetic effects and absence of common litter effects or maternal genetic effects. However, in this study pre-weaning daily gain was influenced by the genes of the individual and the sows as well as common litter effects. The estimates of direct heritability and litter effects for $\mathrm{DG}_{0-8}$ of 0.46 and 0.15 reported in this study suggest the existence of an environmental influence of the sow's ability to take care of the piglet and her milk producing ability. According to these results piglet growth from the same litter resembled each other through their common environment and common genes. The estimate of litter effects in this study is comparable to an estimate of 0.20 for weight gain from three weeks to 18 weeks of age obtained for Australian pigs (Hermesch et al., 2000a) but higher than what has been reported for the Mukota breed in Zimbabwe (Chimonyo \& Dzama, 2007).

Estimates of genetic and phenotypic correlations among various piglet growth traits are presented in Table 4. Genetic correlations ranged from 0.45 to 0.98 while phenotypic correlations ranged from 0.54 to 0.95. Contrary to expectations, there was no tendency of genetic and phenotypic correlations to decrease as the distance between weight measurements increased. The observed inconsistence in genetic and phenotypic 
correlations could be attributed to the variation in the number of records (Table 1). Estimates of genetic and phenotypic correlations among individual piglet weights in developing countries are scarce in the literature.

Prospects to improve performance of various growth traits through selection exist. However, it is important to note that while heavier pigs would be desirable, caution should be exercised not to compromise on carcass quality traits as a result of the correlated response arising from selection for body weight. Therefore, selection strategies that consider both growth performance and carcass quality traits should be considered in future breeding programmes that would match the ever increasing consumer requirements for lean meat. Other traits such as sow reproduction and piglet survival which are major components of the economic efficiency of pig production (Crump et al., 1997b; Hermesch et al., 2000a) should also be included in future pig improvement programmes in Kenya. This would improve on the general performance and economic efficiency of pig production enterprises.

Table 4 Genetic and phenotypic correlation ${ }^{\mathrm{a}}$ among body weight measures

\begin{tabular}{|c|c|c|c|c|c|c|}
\hline Trait $^{\mathrm{b}}$ & BW0 & BW3 & BW8 & BW12 & BW24 & BW36 \\
\hline BW0 & & 0.65 & 0.45 & 0.58 & 0.75 & 0.48 \\
\hline BW3 & 0.70 & & 0.84 & 0.98 & 0.97 & 0.95 \\
\hline BW8 & 0.54 & 0.83 & & 0.85 & 0.86 & 0.93 \\
\hline BW12 & 0.62 & 0.92 & 0.87 & & 0.96 & 0.97 \\
\hline BW24 & 0.75 & 0.92 & 0.85 & 0.95 & & 0.93 \\
\hline BW36 & 0.50 & 0.88 & 0.83 & 0.91 & 0.91 & \\
\hline
\end{tabular}

${ }^{\mathrm{a}}$ Genetic (above diagonal) and phenotypic (below diagonal) correlations.

${ }^{\mathrm{b}}$ See Table 1 for description of traits.

Genetic parameters have been presented using conventional univariate and multivariate models. However, piglet growth traits are measured repeatedly over time. Furthermore, pig breeding programmes in developing countries are characterised by inefficient recording systems and poor data collection. In such cases, random regression models (RRM) would be more robust than conventional models in evaluation of piglet growth performance (Meyer \& Hill, 1997; Huisman et al., 2002). Wasike et al. (2007) evaluated growth of Kenya Boran cattle using RRM and concluded that RRM have potential for modelling growth, notwithstanding conditions of small herd sizes and inconsistent recording. Future research should explore the use of RRM in genetic evaluation of pig growth data. Randon regression models provide an efficient method of evaluating traits that change with time due to their ability to account for the temporal variation in addition to allowing for data interpolation and efficiency of data utilisation.

\section{Conclusions}

This study has shown that early piglet growth is highly heritable and under the influence of maternal genetic and litter effects. If high and sustainable genetic progress is to be guaranteed, selection programmes for genetic improvement of early growth performance should be based on models where direct and maternal genetic effects have been accounted for. This would also be expected to result in genetic improvement in post-weaning growth performance. However, reliable estimation of direct and maternal genetic parameters requires data with a sufficient amount of reliable records and good pedigree structure. This was a major limitation in this study.

\section{Acknowledgements}

The study was supported by the Kenya Agricultural Productivity Project (KAPP). We thank the staff of the Pig Research Unit for their help in data recording and for taking care of the animals. Computing facilities were provided by the Animal Genetic Resources Group, National Animal Husbandry Research Centre, Naivasha, Kenya. 


\section{References}

Bourdon, R.M., 1998. Shortcomings of current genetic evaluation systems. J. Anim. Sci. 76, 2308-2323.

Chimonyo, M. \& Dzama, K., 2007. Estimation of genetic parameters for growth performance and carcass traits in Mukota pigs. Animal 1, 317-323.

Clutter, A.C. \& Brascamp, E.W., 1998. Genetics of performance traits. In: The Genetics of the Pig. Eds. Rothschild, M.F. \& Ruvinsky, A., CAB International, New York. pp. 427-462.

Crump, R.E., Harley, C.S., Thompson, R. \& Mercer, J., 1997a. Individual animal model estimates of genetic parameters for reproductive traits of Landrace Pigs performance tested in a commercial nucleus herd. Anim. Sci. 65, 285-290.

Crump, R.E., Thompson, R., Haley, C.S. \& Mercer, J., 1997b. Individual animal model estimates of genetic correlations between performance test and reproduction traits of Landrace pigs performance tested in a commercial nucleus herd. Anim. Sci. 65, 291-298.

Ehlers, M.J., Mabry, J.W., Bertrand, J.K. \& Stalder, K.J., 2005. Variance components and heritability for sow productivity traits estimated from purebred versus crossbred sows. J. Anim. Breed. Genet. 122, 318-324.

Githinji, M.G., Muasya, T.K., Ilatsia, E.D., Murage, A.W. \& Migose, S.A., 2007. Pig production characteristics and constraints. A case study among smallholder farmers in four districts of Kenya. In: Proceeding of the Animal Production Society of Kenya Annual Scientific Conference, $15^{\text {th }}$ to $16^{\text {th }}$ March 2007, Mtwapa, Kenya.

Hermesch, S., Luxford, B.G. \& Graser, H.U., 2000a. Genetic parameters for lean meat yield, meat quality, reproductive and feed efficiency traits for Australian pigs. 3. Genetic parameters for reproductive traits and genetic correlation with productive, carcass and meat quality traits. Livest. Prod. Sci. 65, 261-270.

Hermesch, S., Luxford, B.G. \& Graser, H.U., 2000b. Genetic parameters for lean meat yield, meat quality, reproductive and feed efficiency traits for Australian pigs. 1. Description of traits and heritability estimates. Livest. Prod. Sci. 65, 239-248.

Huisman, A.E., Veerkamp, R.F. \& Van Arendonk, J.A.M., 2002. Genetic parameters for various random regression models to describe the weight data of pigs. J. Anim. Sci. 80, 575-582.

Johnson, Z.B., Chewning, J.J. \& Nugent, R.A., 2002. Maternal effects on traits measured during postweaning performance test of swine from four breeds. J. Anim. Sci. 80, 1470-1477.

Kabare, N., 1991. Genetic and environmental aspects of reproductive performance and pre-weaning growth rate of three pig breeds in large-scale herds in Kenya. MSc. thesis, University of Nairobi, Kenya.

Kahi, A.K., Rewe, T.O. \& Kosgey, I.S., 2006. Sustainable community-based organisation for the genetic improvement of livestock in developing countries. Outlook Agric. 34, 261-270.

Maniatis, N. \& Pollot, G.E., 2003. The impact of data structure on genetic (co) variance components of early growth traits in sheep, estimated using an animal model with maternal effects. J. Anim. Sci. 81, 101-108.

Meyer, K., 1989. Restricted maximum likelihood to estimate variance component for animal models with several random effects using a derivative free algorithm. Genet. Sel. Evol. 21, 318-327.

Meyer, K., 1992. Variance component due to direct and maternal effects for growth traits of Australian beef cattle. Livest. Prod. Sci. 31, 179-204.

Meyer, K. \& Hill, W.G., 1997. Estimation of genetic and phenotypic covariance functions for longitudinal or 'repeated' records by restricted maximum likelihood. Livest. Prod. Sci. 47, 184-200.

Mungate, F., Dzama, K., Mandisodza, K. \& Shoniwa, A., 1999. Some non-genetic factors affecting commercial pig production in Zimbabwe. S. Afr. J. Anim. Sci. 29, 164-173.

Pathiraja, N., 1987. Improvement of pig-meat production in developing countries. 2. Selection schemes. World Anim. Rev. 61, 2-10.

SAS, 1998. Statistical Analysis System Language Guide for Personal Computers, SAS Institute Inc., Carey, N.C. USA.

Solanes, F.X., Grandinson, K., Rydhmer, L., Stern, S., Andersson, K. \& Lundeheim, N., 2004. Direct and maternal influences on the early growth, fattening performance, and carcass traits of pigs. Livest. Prod Sci. 88, 199-112. 
Solió, L., Rodriguez, M.C., Toro, M.A. \& Rodrigáńez, J., 1994. Maternal and individual effects on piglet weight. In. Proceedings of the $5^{\text {th }}$ World Congress on Genetics Applied to Livestock Production, Guelph, Canada. 17, 355-358.

Wanjaiya, J.K., 1999. Pig production and marketing survey. Proceedings of Pig Stake-holders Workshop, 25-26 February, Egerton University, Njoro, Kenya. p. 4.

Wasike, C.B., Indetie, D., Pitchford, W.S., Ojango, J.M.K \& Kahi, A.K., 2007. Genetic evaluation of growth of Kenya Boran cattle using random regression models. Trop. Anim. Hlth. Prod. 39, 493-505.

Zhang, S., Bidanel, J.P., Burlot, T., Legault, C. \& Naveau, J., 2000. Genetic parameters and genetic trends in the Chinese x European Tiemeslan composite pig line: I. Genetic parameters. Genet. Sel. Evol. 32, 41-56. 